Journal of The Korean Astronomical Society

43: $115 \sim 121,2010$ August

(C)2010 The Korean Astronomical Society. All Rights Reserved.

\title{
NO OPEN CLUSTER IN THE RUPRECHT 93 REGION
}

\author{
Sora Cheon ${ }^{1}$, Hwankyung Sung ${ }^{1}$, And M. S. Bessell ${ }^{2}$ \\ ${ }^{1}$ Department of Astronomy and Space Science, Sejong University, 98 Kunja-dong, Kwangjin-gu, Seoul 143-747, \\ Korea \\ E-mail: sungh@sejong.ac.kr \\ 2 Research School of Astronomy and Astrophysics, Australian National University, MSO, Cotter Road, Weston, \\ ACT 2611, Australia \\ E-mail: bessell@mso.anu.edu.au \\ (Received June 16, 2010; Revised June 28, 2010; Accepted June 29, 2010)
}

\begin{abstract}
$U B V I$ CCD photometry is obtained for the Ruprecht $93(\mathrm{Ru} 93)$ region. We are unable to confirm the existence of an intermediate-age open cluster in $\mathrm{Ru} 93$ from the spatial distribution of blue stars. On the other hand, we find two young star groups in the observed field: the nearer one (Ru 93 group) comprises the field young stars in the Sgr-Car arm at $d \approx 2.1 \mathrm{kpc}$, while the farther one (WR 37 group) is the young stars around WR 37 at $d \approx 4.8 \mathrm{kpc}$. We derive an abnormal extinction law $\left(R_{V}=3.5\right)$ in the Ruprecht 93 region.
\end{abstract}

Key words : color-magnitude diagrams (H-R diagram) - open clusters and associations - stars: early type

\section{INTRODUCTION}

Open clusters can provide valuable information about the formation and evolution of individual stars and stellar systems, the spiral structure of the Galaxy, and the chemical evolution of the Galaxy. Most young open clusters are distributed in the Galactic plane and it is therefore very difficult to select members of the clusters due to foreground and background interlopers. In addition, the small number of member stars and the irregular shape of open clusters make it difficult to test stellar evolution theory and to compare their distribution with the results obtained from dynamical evolution models (Sung et al. 1999).

There are more than 1300 known objects identified as open clusters in the Galaxy. However many of them have still not been observed even in the $U B V$ system (Mermilliod \& Paunzen 2003). In addition, many have not yet been examined critically to see whether they are really open clusters. Ruprecht 93 (Ru 93) was catalogued as an intermediate-age open cluster near $\eta$ Carinae by Ruprecht who later (Ruprecht 1966) classified the cluster as a Trumpler type III 2 with two chains of stars. Steppe (1977) observed 93 stars in the cluster in the $R G U$ photographic photometric system. They determined a distance $(d=1.57 \mathrm{kpc})$ and estimated the earliest spectral type (b7) in Ru 93. The photometric study by Steppe (1977) is the only photometric study for $\mathrm{Ru} 93$ to date. The existence of Wolf-Rayet (WR) star WR 37 near Ru 93 could imply a younger age for $\mathrm{Ru} 93$. From a photometric study of the Ru 93 region

Corresponding Author: H. Sung we have carefully examined the nature of Ru 93 and studied the possible link between Ru 93 and WR 37 .

In Section 2, we present the photometric data for 24,246 stars in the region of $\mathrm{Ru} 93$. In addition, we have identified the 2MASS counterpart of these stars. In Section 3 we derive the reddening law in the region using the intrinsic color relations of early type stars in $U B V I$ and near-IR 2MASS $J H K_{s}$. From the distribution of reddening and the distance modulus of young stars, we have identified two young star groups in the observed region. The age of these young star groups has also been estimated. We make some notes on WR 37, and O9 star HD 305916 in Section 4. Section 5 is the conclusion.

\section{OBSERVATIONS AND DATA REDUC- TION}

$U B V I$ CCD photometry for $\mathrm{Ru} 93$ was performed on 1997 June 23 using the 1-m telescope at Siding Spring Observatory. During the observing run we observed SAAO E region standards (E5 and E7). The standard transformation relations are discussed in Sung \& Bessell (2000), however some coefficients have been modified to avoid the small, non-linear corrections found in the $I, B$ and $U$ transformations. The details of these modifications will be discussed in a forthcoming paper. The atmospheric extinction coefficients were $0.069( \pm 0.009), 0.141( \pm 0.014), 0.277( \pm 0.009)$, and $0.520( \pm 0.016)$ in $I, V, B$, and $U$, respectively. The secondary extinction coefficients in $B$ and $U$ from Sung $\&$ Bessell (2000) have been adopted and used. 
Table 1.

Photometric data ${ }^{a}$

\begin{tabular}{|c|c|c|c|c|c|c|c|c|c|c|c|c|c|c|}
\hline ID & $\alpha(J 2000.0)$ & $\delta(J 2000.0)$ & $V$ & $V-I$ & $B-V$ & $U-B$ & $\epsilon_{V}$ & $\epsilon_{V-I}$ & $\epsilon_{B-V}$ & $\epsilon_{U-B}$ & $\mathrm{n}_{o b s}$ & 2MASS & Sp type & Remark \\
\hline 23913 & $11: 05: 31.19$ & $-61: 17: 27.4$ & 9.253 & - & 1.062 & 0.819 & 0.005 & - & 0.006 & 0.008 & 101 & $11053117-6117271$ & & \\
\hline 10681 & $11: 04: 02.77$ & $-61: 22: 02.1$ & 9.446 & - & 1.667 & 1.562 & 0.007 & - & 0.009 & 0.008 & $\begin{array}{lll}1 & 0 & 1\end{array}$ & $11040280-6122020$ & & \\
\hline 23255 & $11: 05: 26.40$ & $-61: 26: 04.2$ & 9.669 & 0.247 & 0.123 & -0.684 & 0.005 & 0.013 & 0.009 & 0.008 & 111 & $11052637-6126041$ & B0.5III & [ HD 96355 \\
\hline 803 & $11: 02: 48.36$ & $-61: 13: 51.6$ & 9.812 & 0.128 & 0.098 & -0.507 & 0.014 & 0.027 & 0.016 & 0.014 & 111 & $11024831-6113512$ & & \\
\hline 4472 & $11: 03: 15.88$ & $-61: 27: 15.1$ & 9.853 & 0.070 & 0.044 & -0.544 & 0.007 & 0.016 & 0.009 & 0.006 & 111 & $11031589-6127151$ & O9 & HD305916 \\
\hline 19203 & $11: 05: 00.10$ & $-61: 23: 37.0$ & 9.882 & 0.096 & 0.002 & -0.602 & 0.005 & 0.011 & 0.007 & 0.010 & 111 & $11050007-6123369$ & $\mathrm{~B} 2 \mathrm{Ve}$ & HD306016 \\
\hline 11023 & $11: 04: 05.32$ & $-61: 13: 42.5$ & 9.977 & 0.100 & 0.017 & -0.541 & 0.005 & 0.012 & 0.006 & 0.004 & 111 & $11040531-6113427$ & B3III & HD306004 \\
\hline 21415 & $11: 05: 13.93$ & $-61: 20: 41.3$ & 15.126 & 2.242 & 1.161 & 0.548 & 0.003 & 0.004 & 0.004 & 0.015 & 22 & $11051390-6120411$ & WN4 & WR 37 \\
\hline 14185 & $11: 04: 26.22$ & $-61: 25: 45.8$ & 16.303 & 0.421 & 0.214 & -0.600 & 0.010 & 0.021 & 0.020 & 0.021 & 22 & & & WD candidate \\
\hline
\end{tabular}

${ }^{a}$ This is a sample of the full table, which is available from HS.

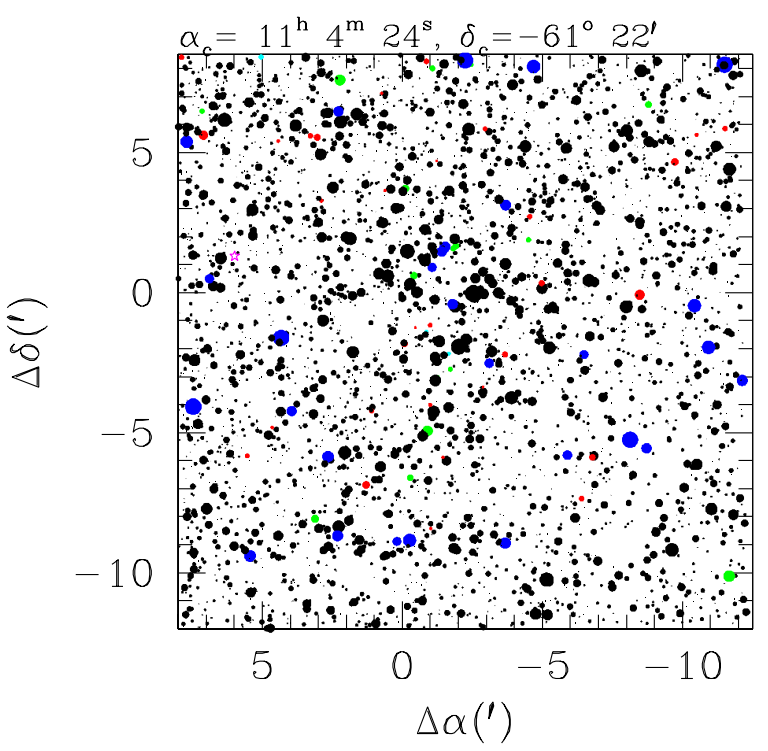

Fig. 1.- Finding chart, centered at $\alpha=11^{h} 4^{m} 24 .{ }^{s} 0, \delta=$ $-61^{\circ} 22 .^{\prime} 0$ (J2000.0). The size of the symbol is proportional to the magnitude of the star. Blue $(E(B-V)=0.2-$ $\left.0.6 \mathrm{mag} \& V_{0}-M_{V} \leq 12 \mathrm{mag}\right)$, green $(E(B-V)=0.25$ $\left.-0.8 \mathrm{mag} \& V_{0}-M_{V}=12-14 \mathrm{mag}\right)$, and red-colored $\left(E(B-V) \geq 1.0 \mathrm{mag} \& V_{0}-M_{V} \leq 14 \mathrm{mag}\right)$ symbols represent, respectively, the young blue stars according to their reddening (see Section 3.2 for details).

The two sets of exposure times for Ru $93-30$ s and $600 \mathrm{~s}$ in $U, 10 \mathrm{~s}$ and $300 \mathrm{~s}$ in $B, 5 \mathrm{~s}$ and $120 \mathrm{~s}$ in $V$, and $5 \mathrm{~s}$ and $60 \mathrm{~s}$ in $I$ - were used to secure both bright and faint stars in the observed field. The point spread function fitting photometric package DAOPHOT implanted in IRAF was used. A total of 24,246 stars were observed in the region. The only saturated star was a K5 star HD $96193(V=7.39)$. Fig. 1 is the finding chart of the $\mathrm{Ru} 93$ region. In order to determine the reddening law in $\mathrm{Ru}$ 93, we have identified the 2MASS counterpart of optical sources. Table 1 shows photometric data for several bright stars, WR 37, and a white dwarf candi- date. We used different colors for different groups in Fig. 1 (see Section 3.2 for the classification of young star groups). We can easily see that there is no clustering of blue stars in the figure. In addition, there is no enhancement of bright blue stars in the presumed core of $\mathrm{Ru} 93$ near $(\Delta \alpha=0.0, \Delta \delta=0.0)$. This fact implies that either there is no open cluster or if there is an open cluster its size is larger than the field of view of our CCD.

\section{PHOTOMETRIC DIAGRAMS AND YOUNG STAR GROUPS IN THE RUPRECHT 93 REGION}

\subsection{Reddening Law}

We present the color-color diagrams from our photometry in Fig. 2. It is very easy to discern that there are at least two groups of reddened blue stars. The median $E(B-V)$ of the least reddened blue stars (filled circles) is about $0.25 \mathrm{mag}$, and that of the highly reddened stars (squares) is about $1.25 \mathrm{mag}$. Several blue stars can be found between these two (triangles). WR 37 (star mark) is fairly red in $(U-B)$. Since there is no intrinsic color relation for WR stars, especially in the broad-band standard photometric systems, due to the contamination by broad emission lines, it is thus impossible to determine the reddening of WR 37. But the colors of WR 37 strongly imply that WR 37 belongs to the more highly reddened group. We also present the $[(B-V)-(V-I),(V-I)]$ diagram in the right panel of Fig. 2 .

Knowledge of the reddening law, especially the totalto-selective extinction ratio $R_{V} \equiv A_{V} / E(B-V)$, is very important in estimating the distance to an object. In general, $R_{V}$ can be determined from the color excess ratio (Guetter \& Vrba 1989). The reddening $E(B-$ $V$ ) of individual early type stars is calculated from the color-color diagram shown in Fig. 2. The color excess of each color is calculated using the relation between intrinsic colors. The intrinsic color relation between $B-V$ and $V-I$ is taken from Sung \& Bessell (1999). Those for 2MASS $J H K_{s}$ were calculated by one of the 

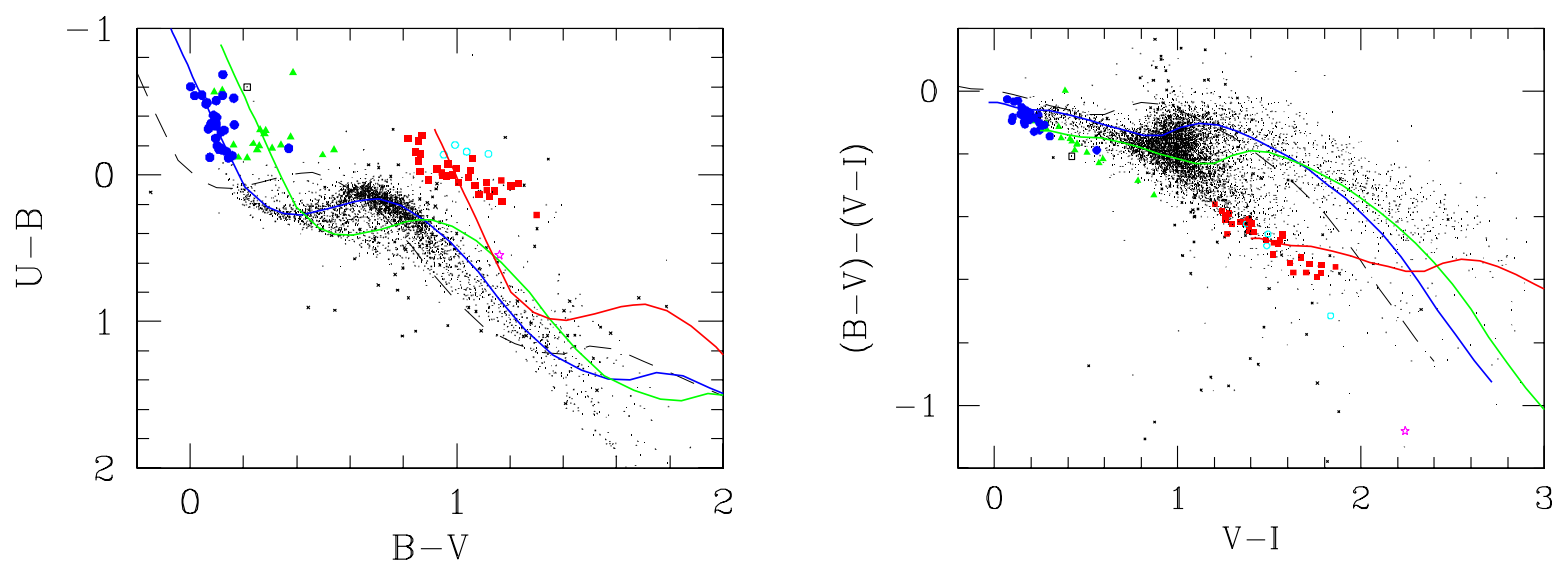

Fig. 2.- Color-Color Diagrams. The thin dashed line represents the ZAMS relation, while the three solid lines represent the ZAMS relations reddened by $E(B-V)=0.25,0.44$, and 1.25 from left to right, respectively. (Blue) Filled circles, (green) triangles, and (red) squares are the stars belonging to these three groups. The star mark (magenta) denotes WR 37 , and a dot within a square represents the white dwarf candidate ID 14185 . The open circles at $B-V \approx 1.0$ show slight differences in photometric characteristics (see Section 3.2 for details). Small dots and crosses represent, respectively, good data $(\epsilon<0.1)$ and bad data $(\epsilon \geq 0.1)$.
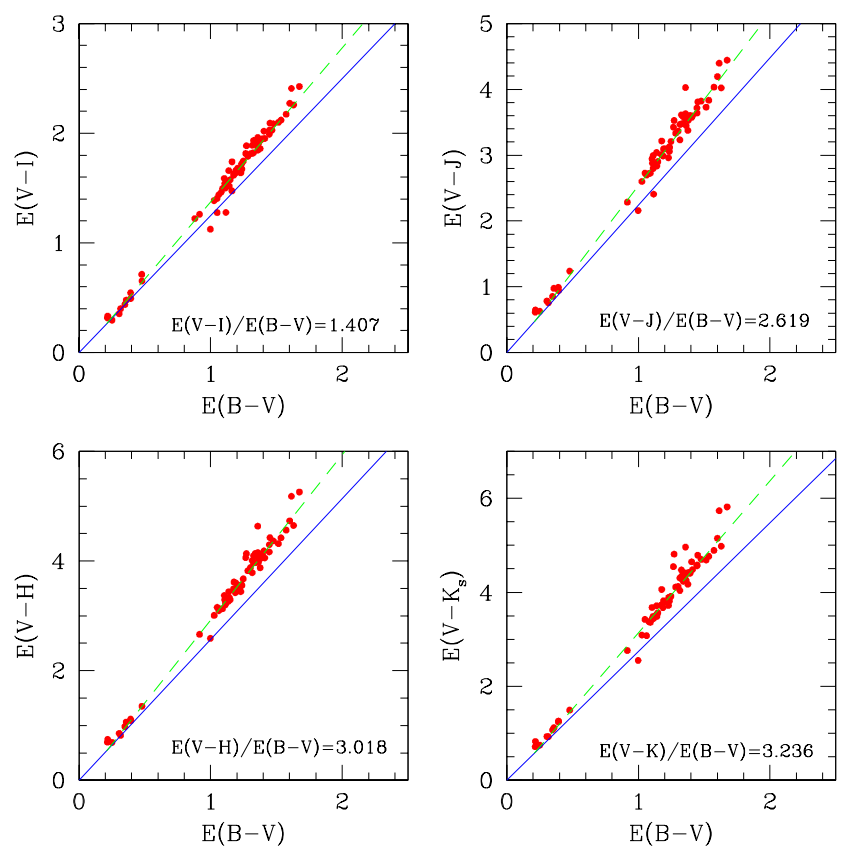

Fig. 3.- Color excess ratios. The color excess of each color is calculated using the relation between intrinsic colors. See text for details. authors (MSB).

Fig. 3 shows the relation between $E(B-V)$ and color excesses in other colors. The thick solid line represents the color excess relation for the normal case, i.e. $R_{V}=3.1$. Evidently early type stars in the $\mathrm{Ru} 93$ region show slightly abnormal values. When we adopt the foreground reddening of $E(B-V)_{f g}=0.22$, the median value for $E(V-I)_{\mathrm{Ru} 93} / E(B-V)_{\mathrm{Ru} 93}, E(V-$ $J)_{\mathrm{Ru} 93} / E(B-V)_{\mathrm{Ru} 93}, E(V-H)_{\mathrm{Ru} 93} / E(B-V)_{\mathrm{Ru} 93}$, and $E\left(V-K_{s}\right)_{\mathrm{Ru}} 9_{3} / E(B-V)_{\mathrm{Ru}} 93$ is $1.407,2.619$, 3.018 , and 3,238 , respectively, where $E(V-C)_{\mathrm{Ru} 93}$ is defined by

$$
\begin{aligned}
& E(V-C)_{\mathrm{Ru} 93} \equiv E(V-C)-E(V-C)_{f g} \\
= & E(V-C)-\left[\frac{E(V-C)}{E(B-V)}\right]_{f g} \cdot E(B-V)_{f g},
\end{aligned}
$$

( $C$ is $I, J, H$, or $K_{s}$ ). We adopt the color excess ratios for the foreground field $[E(V-C) / E(B-V)]_{f g}$ of $1.25,2.24,2.57$, and 2.74 for $I, J, H$, and $K_{s}$, respectively. These values are very similar to those of a $R_{V}=3.1$ star HD 167771 (Fitzpatrick 1999). If we adopt the relation between the color excess ratio and $R_{V}$ of Guetter \& Vrba (1989), the color excess ratios give $R_{V}=3.51 \pm 0.05$ and thus we adopt $R_{V, \mathrm{Ru}} 93=3.5$ for the $\mathrm{Ru} 93$ region.* $^{*}$ 

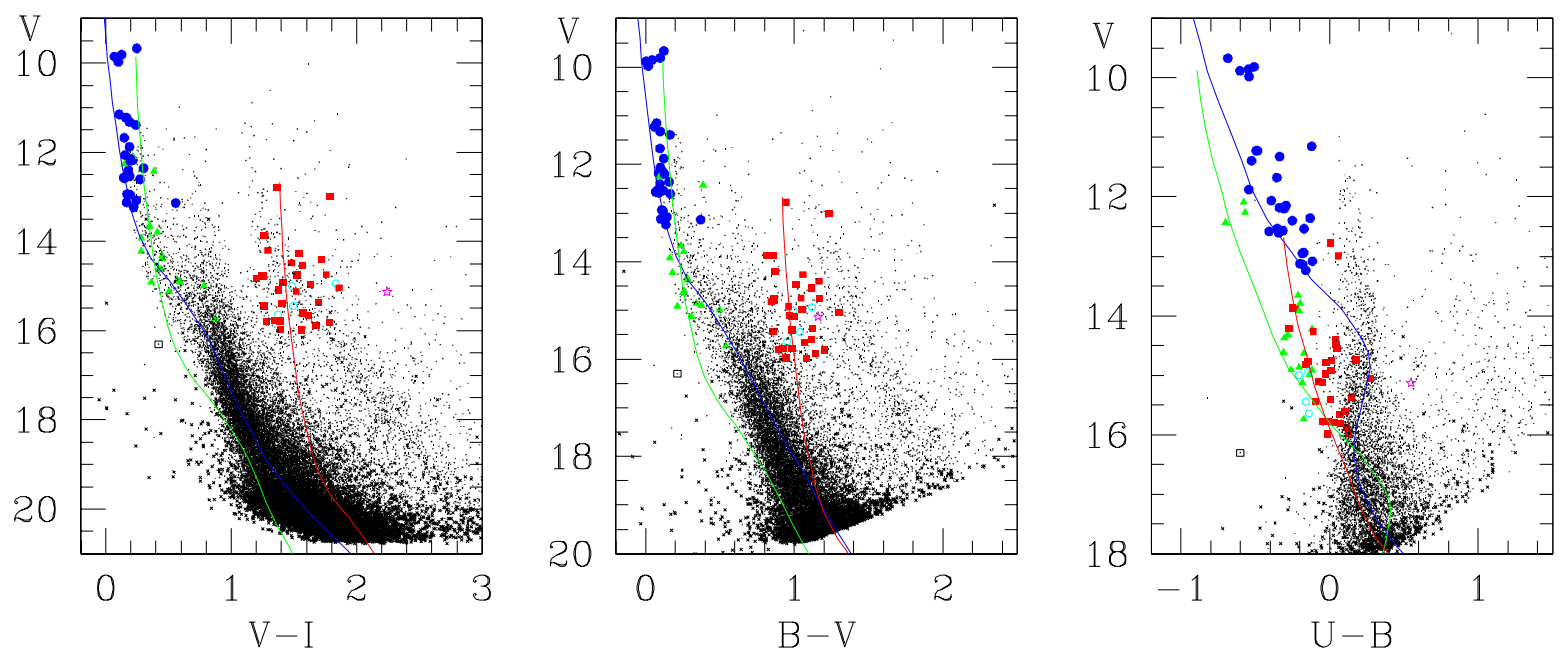

Fig. 4. - Color-Magnitude Diagrams. Three solid lines represent the reddened ZAMS relations reddened by $E(B-V)=$ 0.25 and $V_{0}-M_{V}=11.6, E(B-V)=0.44$ and $V_{0}-M_{V}=13.4$, and $E(B-V)=1.25$ and $V_{0}-M_{V}=13.4$, respectively. All symbols are the same as Fig. 2.

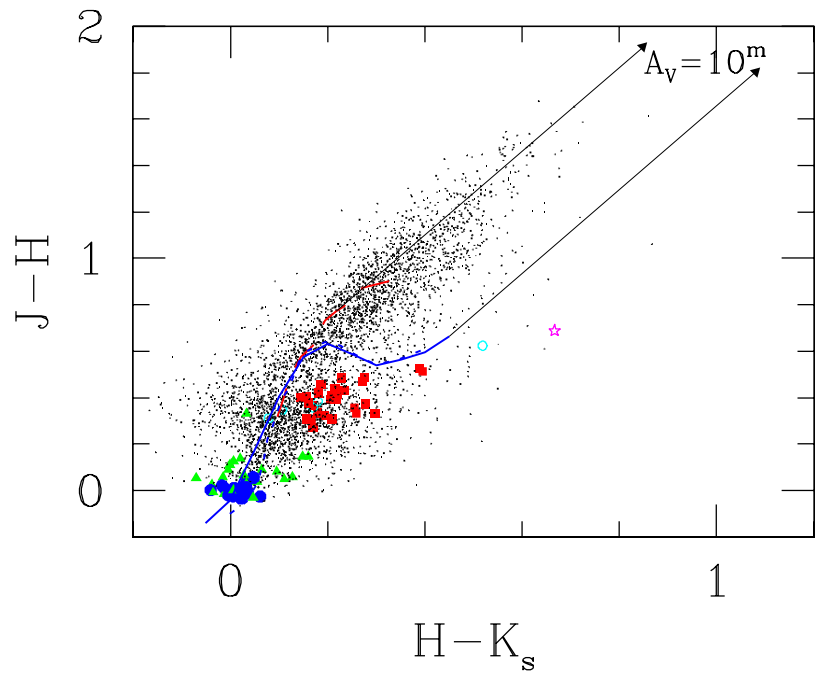

Fig. 5. - Near-IR $J H K_{s}$ color-color diagram for the stars with $\epsilon_{J, H, K_{s}} \leq 0.1$. Two dashed lines are the unreddened relation for MS stars (short dashed line) and giants (long dashed line) from Bessell \& Brett (1988), and the solid line is the unreddened MS relation in 2MASS $J H K_{s}$ from Sung et al. (2008). The thin lines show the direction of the reddening vector of $A_{V}=10$, and the stars between these two lines are reddened normal stars. The other symbols are the same as Fig. 2. The stars ID 20126 (open circle) and WR 37 show an IR excess.

\subsection{Two Young Star Groups}

The distance to an open cluster can be determined using the relation between reddening corrected colors and the absolute magnitudes of zero age main sequence (ZAMS) stars. As mentioned in Section 2, there is no clustering of blue stars in the observed region. This fact implies that it is practically impossible to determine the distance to these stars. Nevertheless, the color-magnitude diagram (CMD) of these blue stars in Fig. 4 is very similar to that of an open cluster. That is because young blue stars can only be in spiral arms as members of OB associations or young open clusters. We calculated the distances to the three blue star groups in Fig. 2. The distance modulus of the less reddened group is about $11.6 \mathrm{mag}(d=2.1 \mathrm{kpc})$. Interestingly, the distance modulus of the other two groups is very similar. ${ }^{\dagger}$ We adopt the distance modulus to the redder groups as $V_{0}-M_{V}=13.4(d=4.8 \mathrm{kpc})$. We present the CMDs in Fig. 4. The reddened ZAMS relations with an appropriate distance modulus are shown in the figure. We call the less reddened group the $\mathrm{Ru}$ 93 group, and the more highly reddened group the WR 37 group.

From Fig. 2 it is seen that the four apparently highly reddened objects having slightly different photometric characteristics. Their distance modulus is further away than the reddened group $\left(V_{0}-M_{V} \approx 14.5\right.$, i.e. $d \approx$ $7.9 \mathrm{kpc})$. But it is also possible to interpret their $(U-$ $B$ ) color as being an UV excess as seen in many classical

of Fitzpatrick (1999) (see their equations A3, A4, and A5), the resulting $R_{V}$ is $3.63 \pm 0.01$.

$\dagger$ Another interpretation for the intermediate reddened group is that they could be pre-main sequence (Herbig $\mathrm{Ae} / \mathrm{Be}$ ) stars associated with the less reddened group. But their numbers are too many and the amount of UV excess is too large when we compare their $(U-B)$ in Fig. 2 with the Herbig Ae/Be star W90 in NGC 2264 (Sung et al. 1997).

\footnotetext{
* If we adopt the relation between the color excess ratio and $R_{V}$
} 

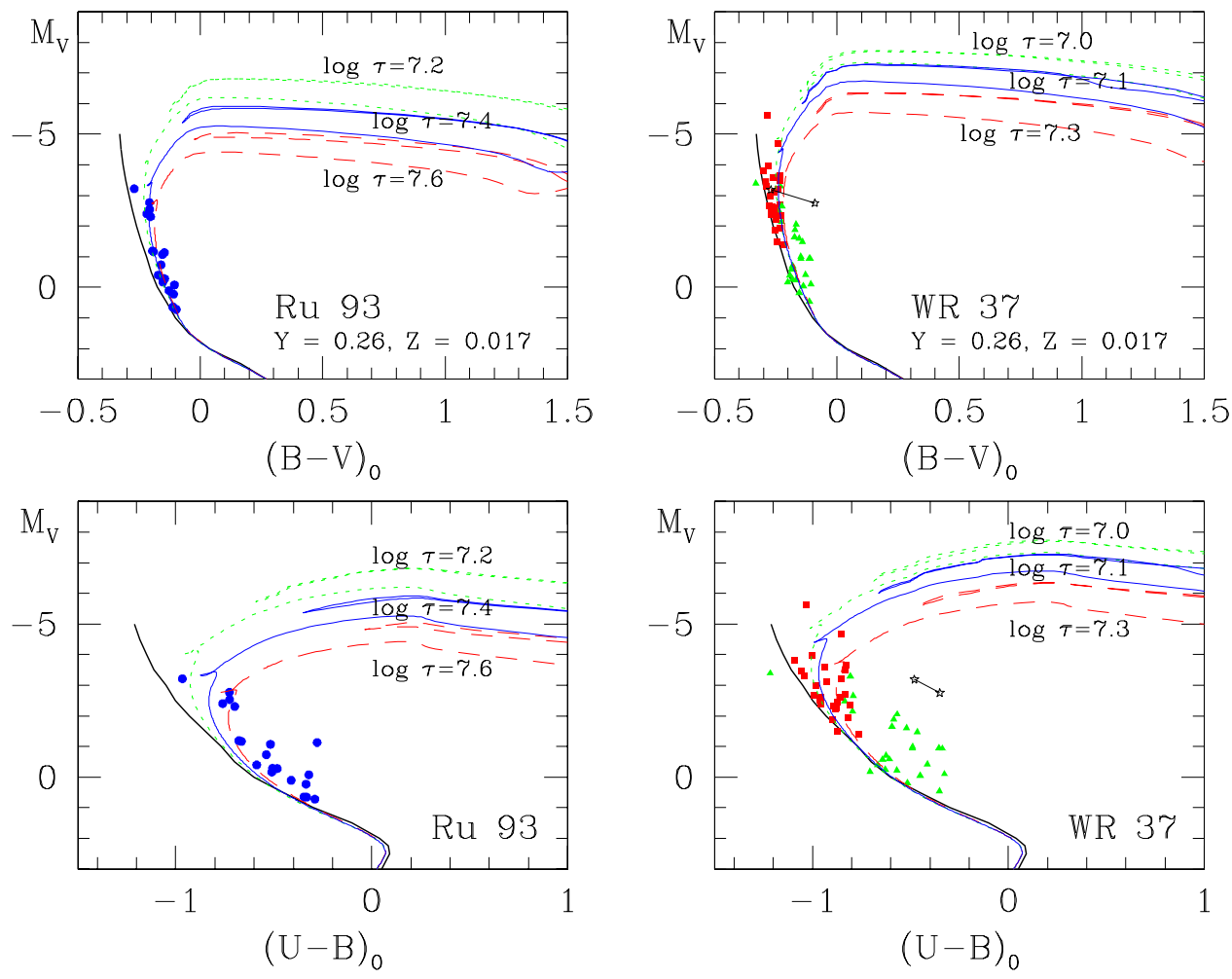

Fig. 6.- H-R diagrams of the Ru 93 and WR 37 groups. The superimposed lines are the isochrones from Bertelli et al. (2009) for solar abundances. The star marks represent the position of WR 37 for $E(B-V)=1.25$ (the median $E(B-V)$ of WR 37 group) and $E(B-V)=1.43$ from van der Hucht (2001).

T Tauri stars. We checked their colors carefully and found that one of them (ID $20126=2$ MASS J110505896113350 ) shows an evident IR excess (see Fig. 5). In addition the star shows excess emission in the $(B-$ $V)-(V-I)$ versus $(V-I)$ diagram in Fig. 2. The star ID 20126 may be a pre-main sequence star in the less reddened group. However, the other three stars do not show any signature of excess emission in the near-IR $J H K_{s}$ or ultraviolet.

The distances to these young star groups are related to the spiral arm structure of the Galaxy. When we compare their location $\left(l=290 .^{\circ} 47, b=-1 . .^{\circ} 11\right)$ in the galactic plane with the distribution of HII regions and giant molecular clouds (see for example Fig. 5 of Hou et al. 2009), their connection with the spiral arms is seen. At $l \approx 290^{\circ}$, the line of sight passes the inner arm twice - once at $d \approx 2.1 \mathrm{kpc}$ and the other at $\approx 7 \mathrm{kpc}$. Two HII regions and a giant molecular cloud can be found between them. The less reddened group is coincident with the near side of the inner spiral arm. The highly reddened group is not coincident with the inner spiral arm but is between the two crossings. It is worth noting however, that the width of a spiral arm is about $500 \mathrm{pc}$ and in addition, the distribution of spiral arm tracers is not well matched with the spiral arm structure of the Galaxy (see Fig. 5 of Hou et al. 2009).

\subsection{Age of Young Star Groups}

As there is no evident clustering of stars in the observed region we cannot derive an age from cluster fitting. However, although there is no known OB association (Humphreys 1978) in the observed region, the blue stars may comprise part of unknown OB associations. The age of young blue stars in the region could represent the age of the youngest population in this region of the spiral arm.

Fig. 6 shows the observational Hertzsprung-Russell (H-R) diagrams of the Ru 93 and WR 37 groups. Superimposed are the most recent isochrones from the Padua group (Bertelli et al. 2009) for solar abundances $(\mathrm{Y}=0.26, \mathrm{Z}=0.017)$. As the solar abundance models of the Padua group are brighter by about 0.3 mag (Sung et al. 1999), the isochrones were shifted vertically by $0.3 \mathrm{mag}$. The age of the $\mathrm{Ru} 93$ group is between $\log \tau=$ 7.2 and 7.6, which is slightly younger than the Pleiades. The age of the WR 37 group is, on the other hand, much younger than the $\mathrm{Ru} 93$ group ( $\log \tau \leq 7.2)$. Several stars in the WR 37 group are smaller than the age of 
the youngest isochrone of Bertelli et al. (2009). If we fit these blue stars with the isochrones of Bertelli et al. (1994) the age of the youngest stars is given by $\log \tau=6.6$. This implies that the star formation in the region around WR 37 occurred very recently (about 4 Myr ago).

\section{INDIVIDUAL STARS}

\subsection{WR 37}

One of main purposes of this study was to estimate the mass of WR 37. As we could not find any young open cluster associated with WR 37, we were unable to constrain the mass and the evolutionary status of WR 37 from cluster age fitting.

As there is no known intrinsic color relation for WR stars in the broad-band photometric system, we cannot estimate the reddening and the intrinsic colors of WR 37. After applying the mean value of reddening, the intrinsic colors of WR 37 are $(V-I)_{0}=0.46,(B-V)_{0}$ $=-0.09,(U-B)_{0}=0.26$, and $M_{V}=-2.75$. Van der Hucht (2001) estimated the reddening to WR 37 as $E_{b-v}=1.43$ from narrow-band photometry, then WR 37 becomes slightly bluer and brighter $\left((V-I)_{0}=\right.$ 0.24, $(B-V)_{0}=-0.27,(U-B)_{0}=-0.48$, and $M_{V}$ $=-3.19)$. He also estimated the distance to WR 37, which is the same value that we obtained for the WR 37 group $\left(V_{0}-M_{v}=13.4\right)$.

\subsection{HD 305916 (=ID 4472)}

HD 305916 (= CPD -60 2483) is classified as O9V (Cruz-Gonzalez et al. 1974). The estimated reddening $E(B-V)=0.25$, and the distance modulus using the ZAMS relation is 9.7 .

If the spectral type of HD 305916 is correct, then the distance modulus of the star becomes $13.5\left(M_{V}(\mathrm{O} 9 \mathrm{~V})\right.$ $=-4.4$ : Sung 1995). In addition, the reddening corrected color of the star should be bluer. If HD 305916 belongs to the WR 37 group, the star is much brighter in Fig. 6. The reddening corrected colors are very similar to those of B type stars. In fact, the spectral type of the star is clearly B3V from a spectrum obtained with WiFeS on the $2.3 \mathrm{~m}$ telescope at SSO on 2010 July 19.

\section{CONCLUSIONS}

$U B V I$ CCD photometry has been obtained for the region around WR 37 , which was previously considered to contain the intermediate-age open cluster $\mathrm{Ru} 93$. Using optical photometry as well as near-IR 2MASS data, we found an abnormal extinction law in the region $\left(R_{V}\right.$, Ru $\left.93=3.5\right)$ and identified two or more groups of blue stars along the line of sight. But we could not find any concentration of blue stars in the observed region. We conclude that $\mathrm{Ru} 93$ is not a real open cluster.
We have determined the distance to the blue stars, and found that the less reddened group of blue stars ( $\mathrm{Ru} 93$ group) is at about $2.1 \mathrm{kpc}$ which coincides with the closest part of the inner arm. The distance to the more highly reddened group (WR 37 group) is about $4.8 \mathrm{kpc}$, which is intermediate between two crossings of the inner spiral arm along the line of sight, and therefore is not well matched with the spiral arm structure of the Galaxy. The age of the young stars in the $\mathrm{Ru}$ 93 group $(\log \tau=7.2-7.6)$ is slightly larger than the young stars in the WR 37 group $(\log \tau \leq 7.2)$.

\section{ACKNOWLEDGMENTS}

H. S. acknowledges the support of the National Research Foundation of Korea (NRF) to the Astrophysical Research Center for the Structure and Evolution of the Cosmos (ARCSEC ${ }^{\prime \prime}$ ) at Sejong University (NRF No. 2009-0062865).

\section{REFERENCES}

Bertelli, G., Nasi, E., Girardi, L., \& Marigo, P. 2009, Scaled Solar Tracks and Isochrones in a Large Region of the Z-Y Plane. II. From 2.5 to $20 \mathrm{M}_{\odot}$ Stars, A\&A, 508, 355

Bertelli, G., Bressan, A., Chiosi, C., Fagotto, F., \& Nasi, E. 1994, Theoretical Isochrones from Models with New Radiative Opacities, A\&AS, 106, 275

Bessell, M. S., \& Bret, J. M. 1988, JHKLM Photometry - Standard Systems, Passbands, and Intrinsic Colors, PASP, 100, 1134

Cruz-Gonzalez, C., Recillas-Cruz, E., Costero, R., Peimbert, M., \& Torres-Peimbert, S. 1974, A Catalogue of Galactic O Stars. The Ionization of the Low Density Interstellar Medium by Runaway Stars, Rev. Mexican A. \& Ap., 1, 211

Fitzpatrick, E. L. 1999, Correcting for the Effects of Interstellar Extinction, PASP, 111, 63

Guetter, H. H., \& Vrba, F. J. 1989, Reddening and Polarimetric Studies toward IC 1805, AJ, 98, 611

Hou, L. G., Han, J. L., \& Shi, W. B. 2009, The Spiral Structure of Our Milky Way Galaxy, A\&A, 499, 473

Humphreys, R. M. 1978, Studies of Luminous Stars in Nearby Galaxies. I. Supergiants and O Stars in the Milky Way, ApJS, 38, 309

van der Hucht, K. A. 2001, The VII-th Catalogue of Galactic Wolf-Rayet Stars, New Astronomy Reviews, 45, 135

Mermilliod, J.-C., \& Paunzen, E. 2003, Analysing the Database for Stars in Open Clusters. I. General Methods and Description of the Data, A\&A, 410, 511

Ruprecht, J. 1966, Classification of Star Clusters, Bull. of Astr. Inst. of Czechslovak, 17, 33 
Steppe, H. 1977, RGU Photometry of Eight Open Clusters near $\eta$ Carinae: NGC 3572, NGC 3590, Tr 18, $\operatorname{Tr} 17, \mathrm{Ru} 92, \mathrm{Ru} 93$, Stock 13 and Ba 17, A\&AS, 27,415

Sung, H. 1995, The UBV CCD Photometry of Eight Young Open Clusters, PhD thesis, Seoul National University

Sung, H., \& Bessell, M. S. 1999, UBVI CCD Photometry of M35 (NGC 2168), MNRAS, 306, 361

Sung, H., \& Bessell, M. S. 2000, Standard Stars - CCD Photometry, Transformation and Comparisons, PASA, 17, 244

Sung, H., Bessell, M. S., \& Lee, S.-W. 1997, UBVRI $\mathrm{H} \alpha$ Photometry of the Young Open Cluster NGC 2264, AJ, 114, 2644

Sung, H., Bessell, M. S., Lee, H.-W., Kang, Y. H., \& Lee, S.-W. 1999, UBVI CCD Photometry of M11. II. New Photometry and Surface Density Profiles, MNRAS, 310, 982

Sung, H., Bessell, M. S., Chun, M.-Y., Karimov, R., \& Ibrahimov, M. 2008, The Initial Mass Function and Young Brown Dwarf Candidates in NGC 2264. III. Photometric Data, AJ, 135, 441 